\title{
Rings, Igloos, and Pebbles of Salt Formed by Drying Saline Drops
}

\author{
Bongsu Shin, ${ }^{\dagger}$ Myoung-Woon Moon, ${ }^{\dagger}$ and Ho-Young Kim ${ }^{*}{ }^{\dagger}$ \\ ${ }^{\dagger}$ Department of Mechanical and Aerospace Engineering, Seoul National University, Shinlim 9-Dong, Gwanak-Gu, Seoul 151-744, \\ Korea \\ ${ }^{\ddagger}$ Institute for Multidisciplinary Convergence of Matter, Korea Institute of Science and Technology, Seoul 136-791, Korea
}

ABSTRACT: It is well-known that evaporation of sessile drops with suspended particles like colloids and coffee powders can yield a variety of two-dimensional patterns depending on the particle shapes and internal flow patterns. Here we show that ordered three-dimensional structures can be built via evaporation of saline drops on highly hydrophobic substrates like pristine PP (polypropylene) with micropores and nanostructured low-surface-energy PP. On pristine PP having a high contact angle but a large contact angle hysteresis (CAH) with water, either rings or igloos of salt are formed depending on the salt concentration and evaporation rate. On

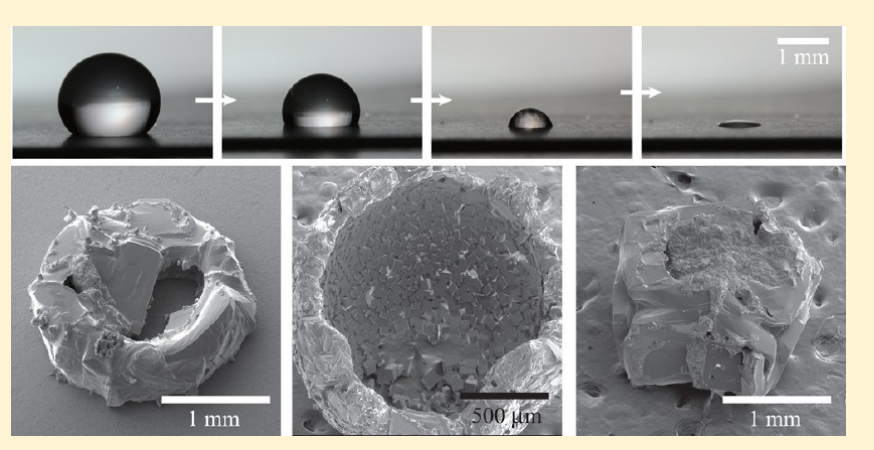
nanostructured low-surface-energy PP having extreme water repellency with a very low $\mathrm{CAH}$, pebbles of salt are formed regardless of salt concentration and evaporation rate. These observations lead us to conclude that combined effects of solubility, evaporation rate, and mobility of the contact line determine the final three-dimensional shape of the salt precipitate.

\section{INTRODUCTION}

Evaporation of sessile drops containing mixed or dissolved solids has implications on various situations ranging from the mundane coffee ring effect ${ }^{1}$ to inkjet printing ${ }^{2}$ and biochip manufacturing. ${ }^{3}$ Since the accumulation of particulate matters along the edge of the drop was explained to be caused by the interior flows replenishing the liquid evaporating at the contact line, various schemes have been proposed to control the deposition patterns of the colloidal suspensions. ${ }^{2,4-6}$ Although two-dimensional structures resulting from self-assembly of those particles like coffee grounds and nanoparticles have been exploited to date, building three dimensionally organized structures through drop evaporation has not been attempted so far.

In this regard, we note that unlike suspensions of particulate matters, drying of some solutions like electrolytes can lead to precipitation and crystallization of salts, which can then be spontaneously ordered to form three-dimensional structures. Nevertheless, the precipitation behavior of saline drops on solid surfaces has been treated far more scarcely than that of mixture drops. The stain morphologies resulting from drying of drops containing sodium chloride $(\mathrm{NaCl})$ on hydrophilic and moderately hydrophobic glass surfaces were investigated by Takhistov and Chang. ${ }^{7}$ It was found that salt was crystallized densely along the edge of the drop for low concentrations while large chunks of salt were formed from a high-concentration drop on hydrophobic surfaces. Drying of aqueous drops of polyelectrolyte and $\mathrm{NaCl}$ was investigated on hydrophilic surfaces, where various two-dimensional patterns such as concentric rings and needle-like structures were found. ${ }^{8} \mathrm{Li}$ et al. reported that the stain pattern resulting from evaporation of a liquid drop with polymeric solutes depends on the contact angle hysteresis. ${ }^{9,10}$

As the previous studies dealing with drying of sessile drops of mixtures of particulate matters and saline solutions have mostly produced two-dimensional structures lying on the substrates, here we aim to show that it is possible to build ordered threedimensional structures through evaporation of sessile saline drops. Reviewing the previous studies hints at a pathway to building three-dimensional structures. First, thin lens-shaped drops cannot produce high-aspect-ratio structures for an obvious reason. Thus, the substrate needs to be highly hydrophobic to prevent spreading of the drop. Second, low salt concentrations naturally lead to deficiency of building materials. High salt concentrations are needed to supply enough materials for three-dimensional architecture.

Here we used highly hydrophobic substrates to start from high-aspect-ratio configurations of sessile drops. Because the mobility of the contact line during drying critically affects the shape evolution of evaporating drops, we employed not only the substrate which pins the contact line but also that which allows the contact line to recede. The solubility of $\mathrm{NaCl}$ in water is slightly over $26 \%$, thus we used saline drops with the concentration ranging from 5 to $20 \%$. In addition to the surface wettability and salt concentration, we changed the evaporation rate to investigate its effects on the precipitation characteristics. In the following, we started with explanation of experimental details and the evaporation behavior of pure water drops sitting

Received: August 2, 2014

Revised: October 2, 2014

Published: October 7, 2014 
on highly hydrophobic surfaces. Then we delineated the evaporation behavior and precipitaion mode of sessile saline drops on surfaces of different wettability and temperature, to show that various three-dimensional structures like rings, igloos, and pebbles can be obtained through evaporation of saline drops on highly hydrophobic surfaces.

\section{MATERIALS AND METHODS}

As solid surfaces on which saline drops sit, we used pristine and modified PP (polypropylene) plates. The pristine PP surface, prepared by hot rolling, possesses numerous micropores of the diameter ranging from approximately 0.5 to $2 \mu \mathrm{m}$ as shown in Figure 1a. To increase the

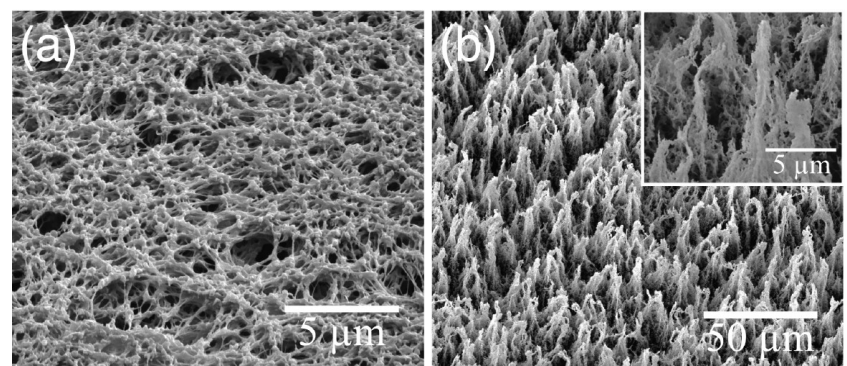

Figure 1. Scanning electron microscopy (SEM) images of (a) pristine $\mathrm{PP}$ and (b) nanostructured superhydrophobic PP.

hydrophobicity of the PP surface, we first etched the PP surface with oxygen plasma to form nanohairy structures. Then the surface was coated with a low-surface-energy material, HMDSO (hexamethyldisiloxane) precursor gas with the surface energy of $23 \mathrm{mN} / \mathrm{m}$, using an rf-PECVD (radio-frequency plasma-enhanced chemical vapor deposition) technique. For detailed process conditions, see Shin et al. ${ }^{11}$ The resulting surface structures are shown in Figure $1 \mathrm{~b}$.

To make saline liquids, we dissolved $\mathrm{NaCl}$ in deionized (DI) water at mass concentrations ranging from 5 to $20 \%$. The solubility of $\mathrm{NaCl}$ in water changes slightly with temperature, so that it varies from 26.4 to $27.5 \%$ as the temperature rises from 20 to $80{ }^{\circ} \mathrm{C}^{12}$ To evaluate the wettability of the PP surfaces, we measured the static and dynamic contact angles of the drops of DI and saline water (5\% and 20\%) at two different substrate temperatures, 20 and $80{ }^{\circ} \mathrm{C}$. For static contact angles, we used drops of $5 \mu \mathrm{L}$ volume on a horizontal surface and measured the angle between the tangents of the liquid-vapor and solid-vapor interfaces at the three-phase contact line. We also measured the contact angle hysteresis ( $\mathrm{CAH})$, the difference between the critical advancing and receding contact angles. The critical advancing (receding) contact angle was measured by increasing (decreasing) the drop volume with an aid of a syringe needle immersed in the drop until the contact line started to move. ${ }^{13}$ Table 1 lists the results of contact angle and $\mathrm{CAH}$ measurements. We see that the static contact angles on each surface hardly varied with the salt concentration while they slightly decreased with the rise of temperature, consistent with the decrease of surface tension of water with temperature. Although the pristine PP exhibited a fairly high static contact angle, $\mathrm{CAH}$ on the surface was very high regardless of salt concentration and temperature. On the nanostructured lowsurface-energy PP surface, the static contact angle exceeded $150^{\circ}$ and $\mathrm{CAH}$ was at most $5^{\circ}$, thus the surface was superhydrophobic.

To record the drying behavior of saline drops, the CMOS (complementary metal oxide semiconductor) camera took the images of the sessile drop every $60 \mathrm{~s}$. As a source of illumination, we used a xenon lamp to image the drop shape and a laser sheet to visualize the motion of salt precipitates inside the drop. The hot plate, on which the PP substrates were located, was controlled to maintain the constant temperature (within $\pm 1{ }^{\circ} \mathrm{C}$ ) as measured by the thermocouple attached to the substrate.

\section{DROP EVAPORATION AND SALT PRECIPITATION}

A. Evaporation of Pure Water Drops. We started with comparing the evaporation behaviors of pure water drops on the two types of surfaces. We imaged and measured the temporal evolutions of the shapes of pure water drops of $5 \mu \mathrm{L}$ in initial volume on the solid surfaces at $20{ }^{\circ} \mathrm{C}$ and present the results in Figure 2. On the pristine PP, a surface with a large $\mathrm{CAH}$, the drop volume shrank with the contact radius held constant in the early stages as shown in Figure 2a,b, which is referred to as the constant-contact-radius (CCR) mode. While the contact line remained pinned, the contact angle decreased down to approximately $110^{\circ}$. Upon the time $t$ reaching about half the entire evaporation duration $t_{\mathrm{f}}$, i.e., at $t / t_{\mathrm{f}} \approx 0.5$, the contact line started to recede while the contact angle was held constant. Here $t_{\mathrm{f}}=70 \mathrm{~min}$. This constant-contact-angle (CCA) mode lasted until $t / t_{\mathrm{f}} \approx 0.8$. Then the contact radius stayed constant again with the rapid decrease of the contact angle until the drop completely dried.

On the nanostructured PP surface with a large contact angle but a very small $\mathrm{CAH}$, Figure $2 \mathrm{c}$,d, the contact angle was kept constant while the contact radius slowly decreased from the beginning until $t / t_{\mathrm{f}} \approx 0.6$, exhibiting the CCA mode. Here $t_{\mathrm{f}}=$

Table 1. Static Contact Angle and CAH of DI and Saline Water Drops on Pristine and Nanostructured Low-Surface-Energy (Superhydrophobic) PP

\begin{tabular}{|c|c|c|c|c|}
\hline surface & temperature $\left({ }^{\circ} \mathrm{C}\right)$ & salt concentration (\%) & static contact angle (deg) & $\mathrm{CAH}(\mathrm{deg})$ \\
\hline \multirow[t]{6}{*}{ pristine PP } & 20 & 0 & $148 \pm 1$ & $79 \pm 10$ \\
\hline & & 5 & $145 \pm 3$ & \\
\hline & & 20 & $150 \pm 2$ & \\
\hline & 80 & 0 & $130 \pm 3$ & $100 \pm 25$ \\
\hline & & 5 & $130 \pm 3$ & \\
\hline & & 20 & $135 \pm 4$ & \\
\hline \multirow[t]{6}{*}{ superhydrophobic PP } & 20 & 0 & $162 \pm 3$ & $3 \pm 2$ \\
\hline & & 5 & $162 \pm 2$ & \\
\hline & & 20 & $161 \pm 2$ & \\
\hline & 80 & 0 & $153 \pm 2$ & $5 \pm 2$ \\
\hline & & 5 & $154 \pm 3$ & \\
\hline & & 20 & $153 \pm 5$ & \\
\hline
\end{tabular}



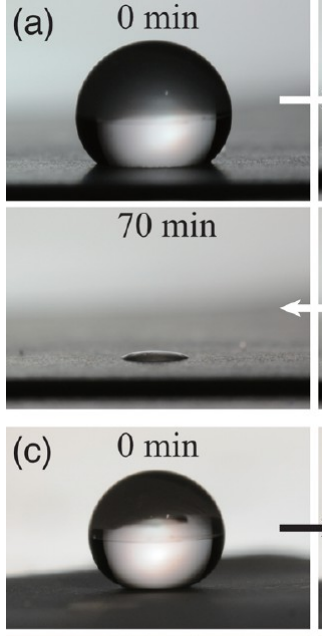

$80 \mathrm{~min}$
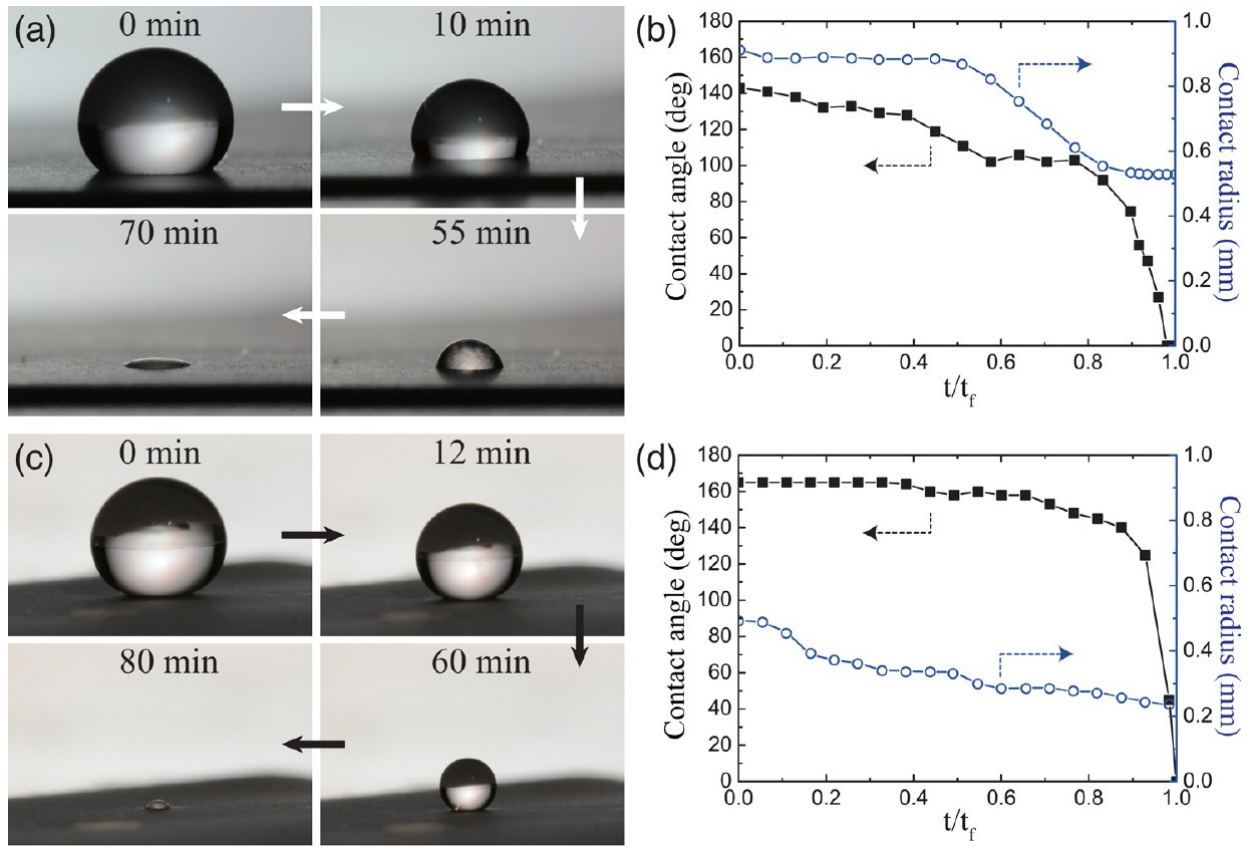

Figure 2. Evaporation behavior of pure water drops of $5 \mu \mathrm{L}$ volume. Optical images (a) and the contact radius and contact angle (b) of a drop on the pristine PP. Optical images (c) and the contact radius and contact angle (d) of a drop on the nanostructured superhydrophobic PP.
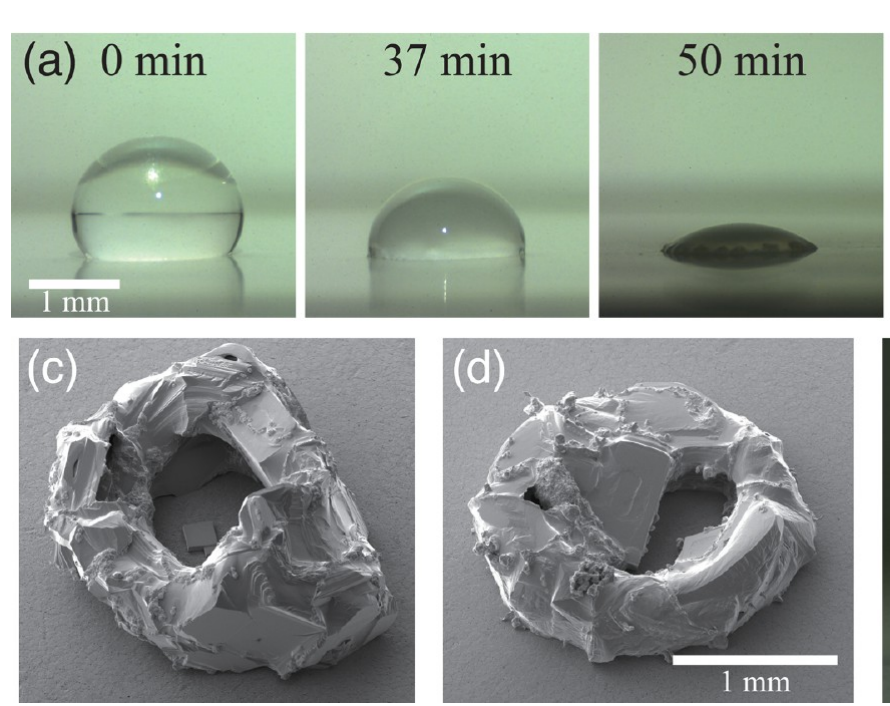
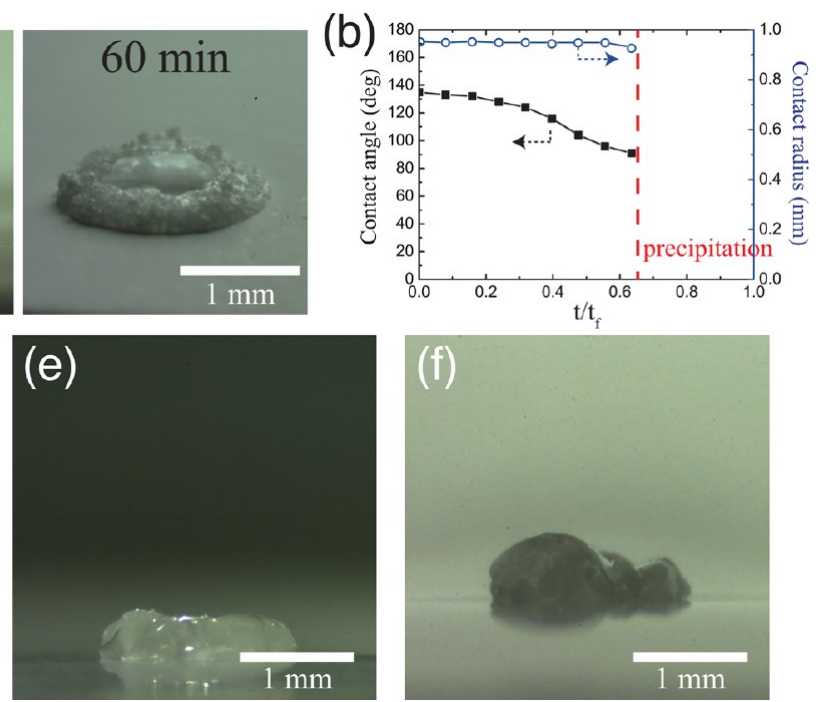

Figure 3. Temporal evolutions of (a) the shape and (b) the contact radius and contact angle of a saline drop of $5 \%$ in initial concentration on the pristine PP surface at $20^{\circ} \mathrm{C}$. The last panel of (a) is a tilted view. In (b), the contact angle and radius are no longer defined beyond $t / t_{\mathrm{f}}=0.65$ when the precipitation occurs. (c) SEM images of the ring of salt. (d) A ring-shaped precipitate formed from a saline drop of 5\% in initial concentration on the pristine PP surface at $80{ }^{\circ} \mathrm{C}$. Salt precipitates formed from saline drops of (e) $10 \%$ and (f) $20 \%$ in initial concentration on the pristine PP surface at $20{ }^{\circ} \mathrm{C}$.

$80 \mathrm{~min}$. Then the contact angle decreased mildly until $t / t_{\mathrm{f}} \approx$ 0.9. In the last stages, $0.9<t / t_{\mathrm{f}}<1$, the contact angle decreased rapidly until the drop vanished. Our experiments confirm the previous reports ${ }^{14,15}$ that the CCA mode prevails on the surfaces of low $\mathrm{CAH}$ while the contact line was pinned to lead to the CCR mode in the beginning of evaporation on the surfaces of high $\mathrm{CAH}$. In the following, we show that different evaporation characteristics, which depend on salt concentrations and substrate temperature as well as surface wettability, can yield different three-dimensional shapes of precipitates when the salt is dissolved in the liquid.

B. Formation of a Ring of Salt. We show the temporal evolution of the morphology of a $5 \%$ saline drop on the pristine
PP surface maintained at $20^{\circ} \mathrm{C}$ in Figure $3 \mathrm{a}$. Figure $3 \mathrm{~b}$ plots the contact radius and contact angle versus time, which reveals that the contact line remains pinned until the average salt concentration reaches the saturation value at $t / t_{\mathrm{f}} \approx 0.65$ where $t_{\mathrm{f}}=60 \mathrm{~min}$. The average salt concentration can be obtained by measuring the drop mass with time assuming no loss of salt during evaporation of water. Optical observation of the drying drop finds the first precipitate at the contact line around $t / t_{\mathrm{f}}=0.65$, consistent with the moment the calculated salt concentration reaches the solubility limit, as shown in the second panel of Figure 3a. The location of precipitation can be understood along the same line as the so-called coffee ring stain formation. As the vapor flux at the contact line was higher than 

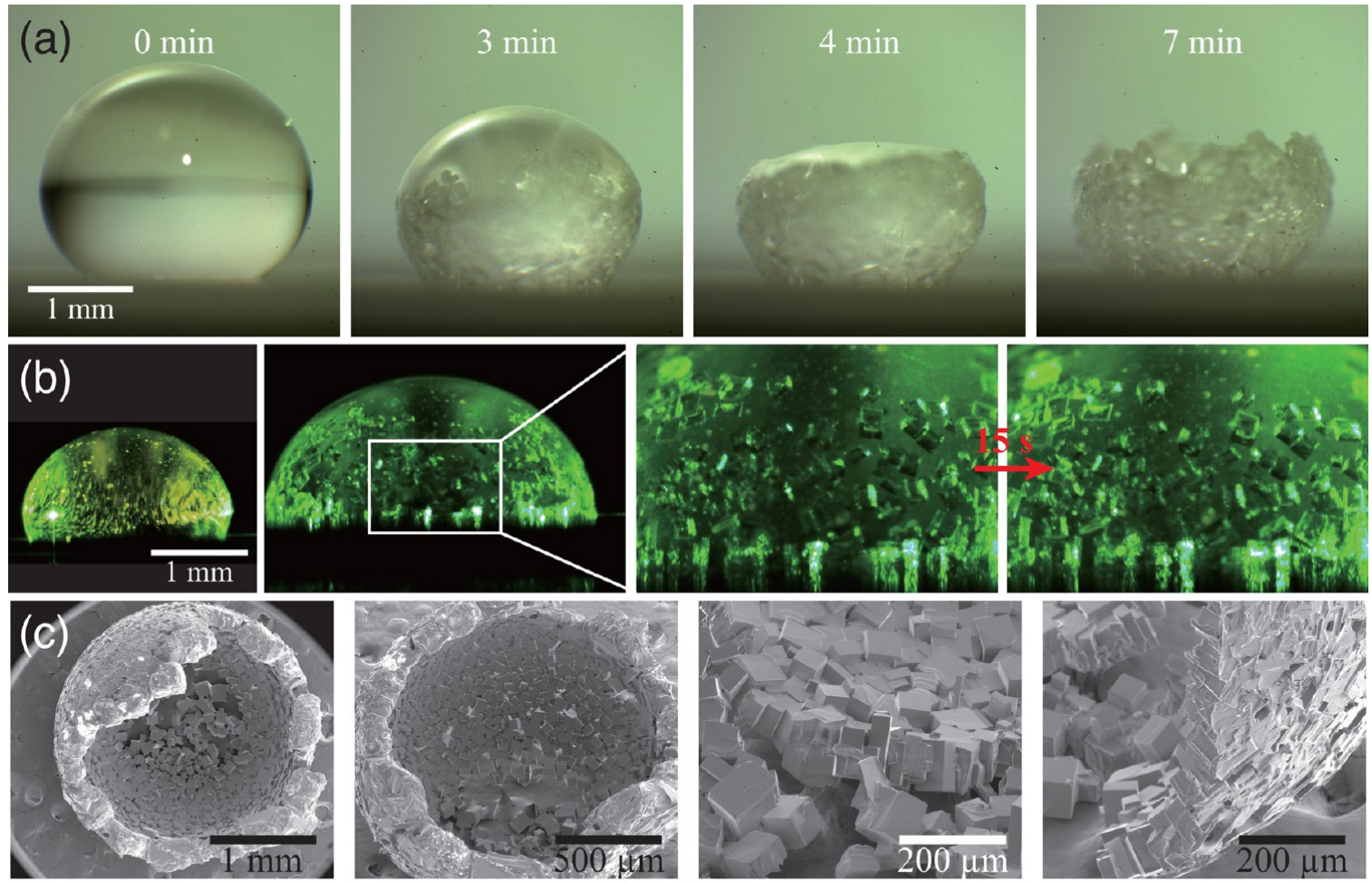

Figure 4. (a) Temporal evolution of a saline drop of $20 \%$ in initial concentration drying on the pristine $\mathrm{PP}$ of $80{ }^{\circ} \mathrm{C}$. (b) Suspended salt cubes just inside the liquid-gas interface illuminated by a laser sheet. (c) SEM images of the salt igloo.

anywhere else on the drop surface when the contact angle was smaller than $\pi / 2{ }^{1}$ the local salt concentration was the highest around the contact line, thus, salt precipitated first near the contact line. Moreover, the compensating flow that keeps the contact line pinned resulted in a continuous supply of solute to the drop edge. Unlike particulate matters suspended in water like coffee powders, $\mathrm{NaCl}$ crystallized to form three-dimensional structures in the vicinity of the contact line, the process of which is displayed in the third and fourth panels of Figure 3a. The result is a ring of salt with a hollow center, Figure $3 \mathrm{c}$. The outer diameter of the ring is found to match the initial contact diameter of the saline drop, $800 \mu \mathrm{m}$ in this case. This is consistent with the hypothesis that the salt precipitation occurs along the initially pinned contact line.

As we increased the evaporation rate of the same concentration (5\%) of saline drops by raising the substrate temperature to $80{ }^{\circ} \mathrm{C}$, the similar precipitation behavior occurred, resulting in the ring of salt, Figure $3 \mathrm{~d}$. This is for an obvious reason that the salt precipitation behavior was unaffected by the evaporation rate at a salt concentration as low as $5 \%$. At higher concentrations ranging from 10 to $20 \%$, the evaporation of saline drops on the substrates of low temperature ranging from 20 to $40{ }^{\circ} \mathrm{C}$ still resulted in ringshaped structures as shown in Figure 3e,f. Increased amount of salt dissolved in the drops led to thicker rims. This indicates that the precipitation still occurs dominantly around the region adjacent to the pinned contact line. At these high concentrations (10-20\%), however, the precipitation behavior changed qualitatively when the evaporation rate was increased, leading to a very different precipitate shape as discussed in Section III.C.

C. Formation of an Igloo of Salt. Figure 4a shows the time sequence of a saline drop of $20 \%$ in initial concentration drying on the pristine PP of $80^{\circ} \mathrm{C}$. Unlike the drops in Figure $3 \mathrm{a}$, the precipitation was not confined within a region near the contact line. Rather, salt precipitated over a wide liquid-gas interfacial region. We found that salt cubes of about $100 \mu \mathrm{m}$ side length were suspended just inside the liquid-gas interface, as shown in Figure $4 \mathrm{~b}$ that uses a thin laser sheet as a lighting. They fell slowly due to gravity and internal circulation and eventually stacked one by one as guided by the drop interface. While the side of the drop was being surrounded by the precipitate layer, water evaporated through the top surface, emptying the inner region. The resulting precipitate resembles an igloo made of ice cubes, which is turned upside down, whose SEM images are displayed in Figure 4c.

The ring in Figure $3 \mathrm{f}$ and the igloo in Figure 4 commonly result from the drying saline drops of $20 \%$ in initial concentration but differ in evaporation rate. The slow evaporation of a high-concentration saline drop continually supplied the liquid and dissolved salt toward the contact line while hardly allowing salt to precipitate elsewhere. This implies that the salt concentration was not high enough to initiate nucleation except for the region near the contact line. On the other hand, precipitation occurred even before the solute was transported to the contact line when the evaporation rate was high, as can be evidenced by the crystallized salt cubes settling slowly at the interface in Figure $4 \mathrm{~b}$.

Although establishing a sophisticated mathematical model to analyze this phase-change phenomena involving convective internal flows and reducing domain size is beyond the scope of this work, here we propose a simple model to understand the thickness of the igloo wall. As schematically shown in Figure 5, precipitation occurred on the drop interface due to water evaporation that generated the flux of water vapor $J$. It was balanced by the diffusive flux of water from inside the drop to 


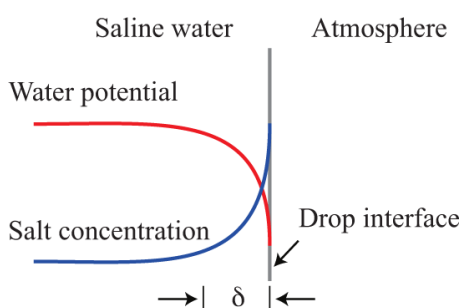

Figure 5. Schematic illustration of the variations of osmotic potential and salt concentration near the interface.

the interface, which can be written as $J \sim \alpha \Delta \Psi / \delta$, where $\Delta \Psi$ is the change of the osmotic potential $\Psi$ across the precipitating layer of the thickness $\delta$ and $\alpha$ is the proportionality constant associated with the diffusion of water molecules in the precipitating layer. The thickness of the precipitating layer is of the same magnitude as the chracteristic distance over which the concentration gradient occurs. The osmotic potential of an ideal solution with a low concentration can be approximated as $\Psi=-i c R T$, where $i$ is the dimensionless van't Hoff factor, $c$ is the molar concentration, $R$ is the gas constant, and $T$ is the absolute temperature. ${ }^{16}$ Although we were dealing with a highconcentration solution, we adopted this relationship for $\Psi$ for the order-of-magnitude analysis. We got $\Delta \Psi \sim 36 \mathrm{MPa}$ with $i=$ $2, c \sim 6.2 \mathrm{~mol} / \mathrm{L}$, corresponding to the salt solubility, $R=8.3 \times$ $10^{3} \mathrm{Nm} / \mathrm{kmol} / \mathrm{K}$, and $T=353 \mathrm{~K}$. Substituting the measured average water flux from the drop, $J=9.2 \times 10^{-4} \mathrm{~kg} \mathrm{~m}^{-2} \mathrm{~s}^{-1}$, leads us to estimate that $\alpha \sim 2.5 \times 10^{-15}$ s to get $\delta \sim 100 \mu \mathrm{m}$, the thickness of the igloo wall.

So far we have shown that both the concentration and the evaporation rate affect the mode of precipitation during evaporation of saline drops with pinned contact lines. Thus, we constructed a regime map based on the experimental results, which allowed us to predict whether rings or igloos will form, as shown in Figure 6. For low concentrations, rings of salt always result regardless of the evaporation rate because of the lack of salt to initiate precipitation anywhere other than the contact line. As the salt concentration increases, igloos tend to form at high evaporation rates (or high substrate temperature). This

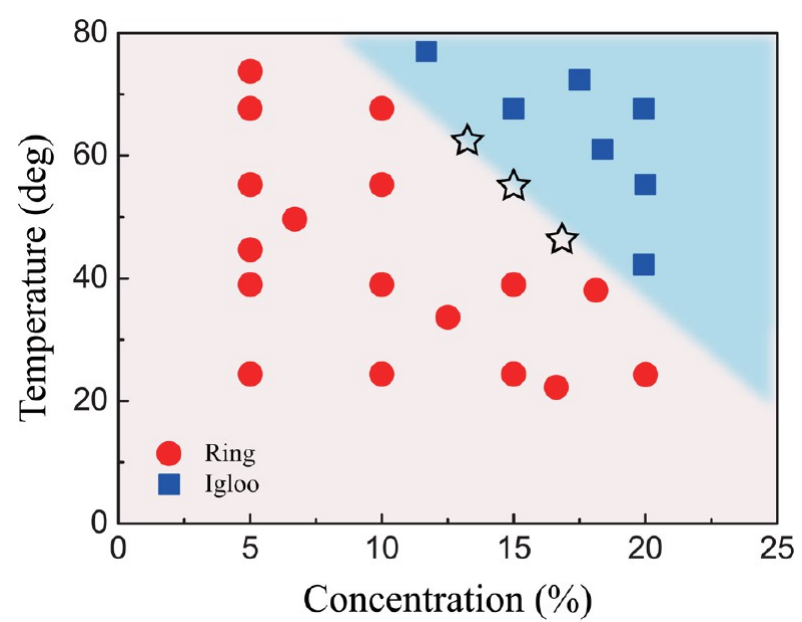

Figure 6. Regime map of precipitation mode on pristine PP based on salt concentration and substrate temperature. The points correspond to the actual experimental results. The stars indicate the conditions where either a ring or an igloo results depending on slight variations in the initial conditions, implying the regime boundary. regime map hints at a possibility that one can design the conditions of saline drops and substrate to control the morphology of the salt precipitate.

D. Formation of a Pebble of Salt. Figure 7a shows the time sequence of a saline drop of $20 \%$ in initial concentration drying on the nanostructured low-surface-energy PP of $80^{\circ} \mathrm{C}$. Experiments of different salt concentrations and substrate temperatures reveal that the salt precipitation behavior on the nanostructured superhydrophobic PP surfaces was little affected by the variations of those parameters in contrast to the evaporation behavior on the pristine PP. The salt precipitate was first observed near the contact line, which is close to the center of the drop bottom, as shown in the first panel of Figure 7a. Salt cubes that formed near the drop interface fell under gravity following the interface then stacked on top of the predeposited chunk of salt, as seen in Figure $7 \mathrm{~b}$. Unstably stacked cubes were sometimes observed to roll down to find a place of lower potential energy by the aid of internally circulating flow indicated as arrows in the first panel of Figure $7 \mathrm{~b}$. The final product of evaporation is a concentrated mass of salt that resembles a pebble, Figure $7 \mathrm{c}$.

We now consider the factors that cause the differences in the precipitate shapes of salt from drops on the pristine $\mathrm{PP}$ and on the nanostructured superhydrophobic PP. First, the drop-solid contact area was very small on the superhydrophobic substrate, and thus all the salt cubes tended to settle toward the center of the drop bottom, facilitating the formation of a single chunk. Second, on the superhydrophobic surface, the salt cubes failed to be aligned along the contact line to form a foundation on which a wall of igloo can be built. This is because the contact line was not pinned on the superhydrophobic surface, which led to weak attachment of the foundation of the precipitate to the superhydrophobic substrate. These factors, combined with the effects of internal circulation that redistribute the salt cubes that were settling on unstable predeposits, resulted in pebbles of salt on superhydrophobic substrates regardless of the salt concentration and evaporation rate.

\section{CONCLUSIONS}

We have experimentally investigated the salt precipitation behavior from saline drops sitting on the hydrophobic PP surfaces at various temperature. On pristine PP surfaces where the contact line is pinned before and during the precipitation process, either rings or igloos of salt are formed depending on the evaporation rate. When the evaporation rate or salt concentration is low, the solute is brought to the contact line, the region of the highest evaporation flux, and then precipitates to make a ring. When the evaporation rate and the salt concentration are high, the solute precipitates on the interface above the contact line and crystallizes to form salt cubes. These suspended cubes slowly settle, guided by the drop interface, which results in a spherical shell consisting of salt cubes having remarkable similarity to an igloo. A regime map has been built that predicts whether a ring or an igloo would be built based on the salt concentration and the substrate temperature. We have further showed that these salt architectures are possible only when the foundation defined by the contact line is solid. On superhydrophobic PP surfaces where the contact line of the very small liquid-solid contact area is highly mobile, salt precipitates cannot be stacked in a regular fashion that would result in either a ring or an igloo. The salt cubes settle toward the center of the drop bottom with the help of gravity and 

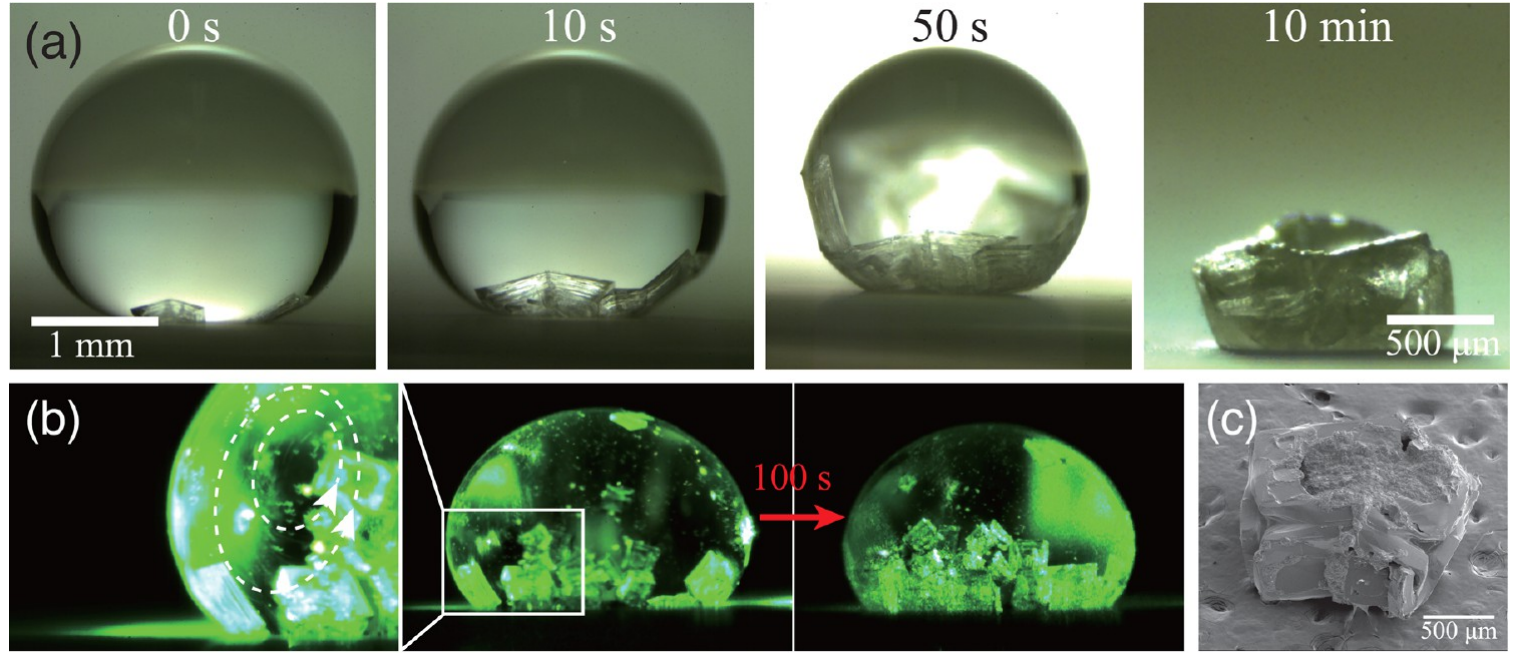

Figure 7. (a) Temporal evolutions of a saline drop of $20 \%$ initial concentration drying on the nanostructured superhydrophobic PP of $80{ }^{\circ} \mathrm{C}$. The same scale bar is used for the first three panels. (b) Suspended salt cubes just inside the liquid-gas interface illuminated by the laser sheet. They are redistributed to attain more stable states by the aid of internal circulation. (c) SEM image of the pebble of salt.

internal circulation, which results in pebbles regardless of the salt concentration and evaporation rate.

Our study can be extended to guide one to predict or even design the shape of precipitates through controlling the concentration of a solution drop and the surface-chemical and thermal states of substrate. While the current work used solutions of $\mathrm{NaCl}$, which yields a cubical structure called halite, other solutes may precipitate in different crystalline structures. Therefore, it is worthy of further study whether evaporation of solution drops of different solutes can lead to different threedimensional precipitate shapes from those reported in this work.

\section{AUTHOR INFORMATION}

\section{Corresponding Author}

*E-mail: hyk@snu.ac.kr.

Notes

The authors declare no competing financial interest.

\section{ACKNOWLEDGMENTS}

This work was supported by KIST, National Research Foundation (grant no. 2013034978), and Ministry of Trade, Industry \& Energy of Korea via SNU-IAMD.

\section{REFERENCES}

(1) Deegan, R. D.; Bakajin, O.; Dupont, T. F.; Huber, G.; Nagel, S. R.; Witten, T. A. Capillary flow as the cause of ring stains from dried liquid drops. Nature 1997, 389, 827-829.

(2) Park, J.; Moon, J. Control of colloidal particle deposit patterns within picoliter droplets ejected by ink-jet printing. Langmuir 2006, 22, 3506-3513.

(3) Dugas, V.; Broutin, J.; Souteyrand, E. Droplet evaporation study applied to DNA chip manufacturing. Langmuir 2005, 21, 9130-9136.

(4) Hu, H.; Larson, R. G. Marangoni effect reverses coffee-ring depositions. J. Phys. Chem. B 2006, 110, 7090-7094.

(5) Weon, B. M.; Je, J. H. Capillary force repels coffee-ring effect. Phys. Rev. E 2010, 82, 015305.

(6) Yunker, P. J.; Still, T.; Lohr, M. A.; Yodth, A. G. Suppression of the coffee-ring effect by shape-dependent capillary interactions. Nature 2011, 476, 308-311.

(7) Takhistov, P.; Chang, H.-C. Complex stain morphologies. Ind. Eng. Chem. Res. 2002, 41, 6256-6269.
(8) Kaya, D.; Belyi, A.; Muthukumar, M. Pattern formation in drying droplets of polyelectrolyte and salt. J. Chem. Phys. 2010, 133, 114905.

(9) Li, Y.-F.; Sheng, Y.-J.; Tsao, H.-K. Evaporation stains: suppressing the coffee-ring effect by contact angle hysteresis. Langmuir 2013, 29, $7802-7811$.

(10) Li, Y.-F.; Sheng, Y.-J.; Tsao, H.-K. Solute concentrationdependent contact angle hysteresis and evaporation stains. Langmuir 2014, 30, 7716-7723.

(11) Shin, B.; Moon, M.-W.; Lee, K.-R.; Kim, H.-Y. Extreme water repellency of nanostructured low-surface-energy non-woven fabrics. Soft Matter 2012, 8, 1817-1823.

(12) Pinho, S. P.; Macedo, E. A. Solubility of $\mathrm{NaCl}, \mathrm{NaBr}$, and $\mathrm{KCl}$ in water, methanol, ethanol, and their mixed solvents. J. Chem. Eng. Data 2005, 50, 29-32.

(13) de Gennes, P.-G.; Brochard-Wyart, F.; Quéré, D. Capillarity and Wetting Phenomena: Drops, Bubbles, Pearls, Waves; Springer: New York, 2004.

(14) Birdi, K. S.; Vu, D. T.; Winter, A. A study of the evaporation rates of small water drops placed on a solid surface. J. Phys. Chem. 1989, 93, 3702-3703.

(15) Song, H.; Lee, Y.; Jin, S.; Kim, H.-Y.; Yoo, J. Y. Prediction of sessile drop evaporation considering surface wettability. Microelectron. Eng. 2011, 88, 3249-3255.

(16) Adamson, A. W.; Gast, A. P. Physical Chemistry of Surfaces, 6th ed.; Wiley: New York, 1997. 